\title{
Coherent approach to transport and noise in double-barrier resonant diodes
}

\author{
V. Ya. Aleshkin \\ Institute for Physics of Microstructures, Nizhny Novgorod GSP-105 603600, Russia \\ L. Reggiani \\ National Nanostructure Laboratory of INFM, Dipartimento di Ingegneria dell' Innovazione, Università di Lecce, \\ Via Arnesano s/n, 73100 Lecce, Italy \\ N. V. Alkeev and V. E. Lyubchenko \\ Institute of Radioengineering and Electronics, Russian Academy of Sciences, Moscow District B-71, Russia \\ C. N. Ironside, J. M. L. Figueiredo, and C. R. Stanley \\ Department of Electronic and Electrical Engineering, University of Glasgow, Glasgow G12 8LT, United Kingdom \\ (Received 15 January 2003; revised manuscript received 22 April 2004; published 23 September 2004)
}

\begin{abstract}
We implement a quantum approach which includes long range Coulomb interaction and investigate current voltage characteristics and shot noise in double-barrier resonant diodes. The theory applies to the region of low applied voltages up to the region of the current peak and considers the wide temperature range from zero to room temperature. The shape of the current voltage characteristic is well reproduced and we confirm that even in the presence of Coulomb interaction the shot noise can be suppressed with a Fano factor well below the value of 0.5 . This feature can be an indication of coherent tunneling since the standard sequential tunneling predicts in general a Fano factor equal to or greater than the value 0.5. This giant suppression is a consequence of Pauli principle as well as long range Coulomb interaction. The theory generalizes previous findings and is compared with experiments.
\end{abstract}

DOI: $10.1103 /$ PhysRevB.70.115321

PACS number(s): 72.70. $+\mathrm{m}, 72.20 .-\mathrm{i}, 72.30 .+\mathrm{q}$

\section{INTRODUCTION}

Since its realization, ${ }^{1}$ the double-barrier resonant diode (DBRD) proved to be an electron device of broad physical interest because of its peculiar non-Ohmic current voltage $(I-V)$ characteristic. Indeed, after a strong superOhmic increase of current it exhibits a negative differential conductance and eventually histeresis effects. ${ }^{2}$ Even the shot noise characteristics of DBRDs are of relevant interest in the sense that suppressed as well as enhanced shot noise with respect to the full Poissonian value has been observed (see Ref. 3 for a review on the subject). These electrical and noise features are controlled by the mechanism of carrier tunneling through the double potential barriers. The microscopic interpretation of these features is found to admit a coherent or a sequential tunneling approach. ${ }^{3}$ The coherent approach ${ }^{4}$ consists in considering the whole device as a single quantum system characterized by a transmission coefficient describing carrier transport from one contact to the other. By contrast, the sequential approach ${ }^{5}$ consists in considering tunneling through the diode as a two step process where carriers first transit from one contact into the well, then from the well to the other contact. The intriguing feature of these two approaches is that from the existing literature it emerges that both of them are capable to explain the $I-V$ experimental data as well as most of the shot noise characteristics. Therefore, to our knowledge there is no way to distinguish between these two transport regimes and the natural question whether the tunneling transport is coherent or sequential remains an unsolved one.

The coherent approach to shot noise in DBRD has received wide attention since the first experimental evidence by Li et $a l .{ }^{6}$ of shot noise suppression with a minimum value of the Fano factor $\gamma=S_{I} /(2 q I)=0.5$, here $S_{I}$ is the low frequency spectral density of current fluctuations and $q$ is the absolute value of the unit charge responsible of current. Remarkably, most of the coherent approaches developed so $\operatorname{far}^{7-12}$ predict a maximum suppression $\gamma=0.5$. On the other hand, there is experimental evidence of suppression below this value, ${ }^{8,13,14}$ down to values of $\gamma=0.25^{13,14}$ which, despite being the result of spurious effects and/or measurements uncertainty, are not yet entirely understood. To this purpose, some authors obtained theoretical values of the Fano factor just below the value of 0.5 , Ref. 15 found 0.45 and Ref. 16 0.38 , respectively. However, the physical interpretation of these results remains mostly qualitative and quoting Ref. 3 this direction of research looks promising but certainly requires more efforts. We remark that the theory of shot noise in DBRDs under the sequential approach ${ }^{17-21,15,11,22-24}$ provides a Fano factor of 0.5 as the minimum value of shot noise suppression.

The aim of this paper is to develop a coherent theoretical approach for current voltage and electronic noise in DBRDs accounting for the Pauli principle and long range Coulomb interaction going beyond existing models. To this purpose, we implement the quantum approach proposed in Refs. $25,22,26$. We anticipate that the main result of the present theoretical approach concerns the prediction that suppression of shot noise with a Fano factor below 0.5 can be an indication of coherent tunneling against sequential tunneling.

The content of the paper is organized as follows. Section II presents the theoretical model. Section III provides the analytical expressions for the calculation of the current volt- 


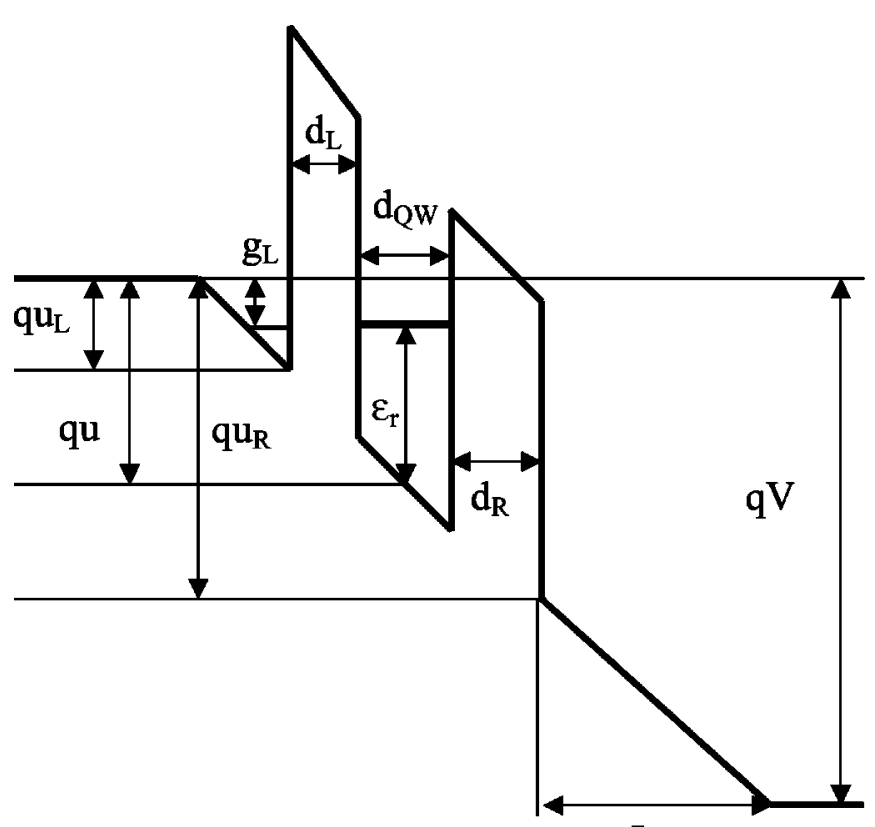

L

FIG. 1. Sketch of the band profile of the double-barrier structure considered here. The bottom of the conduction band in the emitter in the well and in the collector coincides at $V=0$.

age characteristics in the low voltage region limited to the first peak of the current. Section IV provides the analytical expression for the electron noise corresponding to the current voltage characteristics of Sec. III. Here, the Nyquist expression is recovered at vanishing applied voltage. At increasing voltages suppressed shot noise is found in the region preceding the current peak and enhanced shot noise at voltages just above the current peak. Section V reports a comparison between theory and existing experiments. Major conclusions are drawn in Sec. VI.

\section{MODEL}

The typical diode investigated here is the standard double well structure depicted in Fig. 1 . We denote by $\Gamma=\left(\Gamma_{L}\right.$ $\left.+\Gamma_{R}\right)$ the resonant states width, by $\varepsilon_{r}$ the energy of the resonant level as measured from the center of the potential well and by $\Gamma_{L, R}$ the partial widths due to the tunneling through the left and the right barrier, respectively. We consider the case of coherent tunneling in the presence of only one resonant state and we assume that the diode has contacts with unit square surfaces. For convenience, calculations are carried out using the cgs system.

The kinetic model is developed by assuming that the electron distribution functions in the emitter and in the collector, $f_{\alpha}$, are equilibrium-like, but with different electrochemical potentials $F_{\alpha}$ :

$$
f_{\alpha}\left(\varepsilon, F_{\alpha}\right)=\frac{1}{1+\exp \left(\frac{\varepsilon-F_{\alpha}}{k_{B} T}\right)},
$$

here $\alpha=L$ stands for the left contact (the emitter), $\alpha=R$ for the right contact (the collector), $k_{B}$ is the Boltzmann con- stant, $T$ is the bath temperature, and $\varepsilon$ is the carrier energy.

The full Hamiltonian of the structure is

$$
H=H_{L}+H_{R}+H_{\text {res }}+H_{\text {tun }}
$$

here

$$
H_{\alpha}=\sum_{p_{\perp}} \sum_{p_{\alpha}}\left(E\left(p_{\alpha}\right)+p_{\perp}^{2} / 2 m\right) c_{\alpha}^{+}\left(p_{\alpha}, p_{\perp}\right) c_{\alpha}\left(p_{\alpha}, p_{\perp}\right)
$$

are the Hamiltonians of the right and left contacts, $c_{\alpha}^{+}$and $c_{\alpha}$ are the creation and annihilation operators of electrons in contact $\alpha, p_{\perp}$ and $p_{\alpha}$ are the momentum components perpendicular and parallel to the direction of the current, respectively, $m$ is the effective electron mass in the conduction band, and $E\left(p_{\alpha}\right)+p_{\perp}^{2} / 2 m$ is the electron energy in the $\alpha$ contact

$$
H_{\text {res }}=\sum_{p_{\perp}}\left(E_{r}+p_{\perp}^{2} / 2 m\right) a^{+}\left(p_{\perp}\right) a\left(p_{\perp}\right)
$$

is the Hamiltonian of the resonant states, $E_{r}=\varepsilon_{r}-q u$ with $u$ is the voltage drop between the emitter and center of the quantum well, $-q$ is the electron charge, $a^{+}$and $a$ are the creation and annihilation operators of electrons in the resonant level, and

$$
H_{\mathrm{tun}}=\sum_{p_{\perp}, \alpha}\left[T_{\alpha} a^{+}\left(p_{\perp}\right) \sum_{p_{\alpha}} c_{\alpha}\left(p_{\alpha}, p_{\perp}\right)+h c\right]
$$

is the part of the Hamiltonian describing the electron tunneling and $T_{\alpha}$ is the amplitude of tunneling between the resonant state and the $\alpha$ th contact.

Following Ref. 25, the relation between $\Gamma_{\alpha}$ and $T_{\alpha}$ is: $\Gamma_{\alpha}=2 \pi\left|T_{\alpha}\right|^{2} \rho_{\alpha}$ where $\rho_{\alpha}$ is the one dimensional density of states, and the electron operators $a$ and $c$ in the Heisenberg representation are given by

$$
a\left(t, p_{\perp}\right)=\sum_{\alpha, p_{\alpha}} T_{\alpha} \frac{\exp \left\{-i\left[E\left(p_{\alpha}\right)+p_{\perp}^{2} / 2 m\right] t / \hbar\right\}}{E\left(p_{\alpha}\right)-E_{r}+i \Gamma / 2} c_{\alpha}\left(p_{\alpha}, p_{\perp}\right),
$$

$$
\begin{aligned}
c_{\alpha}\left(t, p_{\alpha}, p_{\perp}\right)= & c_{\alpha}\left(p_{\alpha}, p_{\perp}\right) \exp \left\{-i\left[E\left(p_{\alpha}\right)+p_{\perp}^{2} / 2 m\right] t / \hbar\right\} \\
& -\frac{i T_{\alpha}^{*}}{\hbar} \int_{-\infty}^{t} d \tau a\left(\tau, p_{\perp}\right) \\
& \times \exp \left\{-i\left[E\left(p_{\alpha}\right)+p_{\perp}^{2} / 2 m\right](t-\tau) / \hbar\right\} .
\end{aligned}
$$

The current operators for the left and right contacts $I_{L, R}$ and for the total current $I$ are $^{25}$

$$
\begin{gathered}
I_{\alpha}(t)=-\frac{i q}{\hbar} \sum_{p_{\alpha} p_{\perp}}\left[T_{\alpha} a^{+}\left(t, p_{\perp}\right) c_{\alpha}\left(t, p_{\alpha}, p_{\perp}\right)-h c\right], \\
I=\eta I_{L}-(1-\eta) I_{R},
\end{gathered}
$$

where $\eta=u / V, V$ being the total applied voltage (see Fig. 1).

From Eqs. (3)-(5) we obtain the expressions for current operators similar to that obtained in Ref. 22: 


$$
\begin{aligned}
I_{\alpha}(t)= & \frac{q}{2 \pi \hbar} \sum_{p_{\perp}} \sum_{\beta, p_{\beta}} \sum_{\gamma, p_{\gamma}} \exp \left[i\left(E_{\beta}-E_{\gamma}\right) t / \hbar\right] \\
& \times A_{\beta \gamma}^{\alpha}\left(E_{\beta}, E_{\gamma}\right) c_{\beta}^{+}\left(p_{\beta}, p_{\perp}\right) c_{\gamma}\left(p_{\gamma}, p_{\perp}\right),
\end{aligned}
$$

where $E_{\alpha}=E\left(p_{\alpha}\right)$ and the full expression for $A_{\beta \gamma}^{\alpha}\left(E_{\beta}, E_{\gamma}\right)$ is reported in the Appendix. From Eqs. (6) and (7) and the expression of $A_{\beta, \gamma}^{\alpha}\left(E_{\beta}, E_{\gamma}\right)$ from the Appendix, we find the usual expression for the average current

$$
\begin{aligned}
\langle I\rangle & =\left\langle I_{L}\right\rangle=-\left\langle I_{R}\right\rangle \\
& =\frac{q m}{2 \pi^{2} \hbar^{3}} \int_{-g_{L}}^{\infty} d \varepsilon_{z} D\left(\varepsilon_{z}\right) \int_{0}^{\infty} d \varepsilon_{\perp}\left[f_{L}\left(\varepsilon, F_{L}\right)-f_{R}\left(\varepsilon, F_{R}\right)\right],
\end{aligned}
$$

where $\varepsilon_{\perp}=p_{\perp}^{2} / 2 m$ is the kinetic energy of the transverse motion, $\varepsilon=\left(\varepsilon_{z}+\varepsilon_{\perp}\right), g_{L}\left(u_{L}\right)$ is the energy gap between the bottom of the conduction band and the first quantized level in the well before the left barrier (see Fig. 1), $D\left(\varepsilon_{z}\right)$ the transparency of the double barrier given by

$$
D\left(\varepsilon_{z}\right)=\frac{\Gamma_{L} \Gamma_{R}}{\left(\varepsilon_{z}-\varepsilon_{r}+q u\right)^{2}+\Gamma^{2} / 4} .
$$

In our model, we suppose that in the emitter there are no electron states with energy below $-g_{L}$ and that electron states with energy higher than this value are three dimensional.

To take into account Coulomb effects, we introduce the expression for the operator of the electron charge in the quantum well, that from Eq. (3) is found to be given by

$$
\begin{aligned}
Q_{Q W}(t)= & -q \sum_{p_{\perp}} a^{+}\left(t, p_{\perp}\right) a\left(t, p_{\perp}\right) \\
= & -q \sum_{p_{\perp}} \sum_{\alpha, p_{\alpha}} \sum_{\beta, p_{\beta}} \frac{T_{\alpha}^{*} T_{\beta} \exp \left[i\left(E_{\alpha}-E_{\beta}\right) t / \hbar\right]}{\left(E_{\alpha}-E_{r}-i \Gamma / 2\right)\left(E_{\beta}-E_{r}+i \Gamma / 2\right)} \\
& \times c_{\alpha}^{+}\left(p_{\alpha}, p_{\perp}\right) c_{\beta}\left(p_{\beta}, p_{\perp}\right) .
\end{aligned}
$$

From Eq. (9) the average electron charge in the quantum well is found to be

$$
\begin{aligned}
\left\langle Q_{Q W}\right\rangle= & -\frac{q m}{2 \pi^{2} \hbar^{2}}\left[\int_{-g_{L}}^{\infty} d \varepsilon_{z} D\left(\varepsilon_{z}\right) \int_{0}^{\infty} d \varepsilon_{\perp} \Gamma_{R}^{-1} f_{L}\left(\varepsilon, F_{L}\right)\right. \\
& \left.+\int_{-q V}^{\infty} d \varepsilon_{z} D\left(\varepsilon_{z}\right) \int_{0}^{\infty} d \varepsilon_{\perp} \Gamma_{L}^{-1} f_{R}\left(\varepsilon, F_{R}\right)\right] .
\end{aligned}
$$

\section{CURRENT VOLTAGE CHARACTERISTICS}

The current voltage characteristic is determined from Eq. (8) once the dependence of $D\left(\varepsilon_{z}\right)$ and $g_{L}$ on the applied voltage is given. To calculate the transparency explicitly, we must find $u_{L}(V)$ and $u(V)$ as functions of $V$. To this purpose, we consider the Poisson equation for the electrical potential $\varphi$ in the structure of Fig. 1. In the emitter, the Poisson equation can be written in the form

$$
\varphi^{\prime \prime}=\frac{4 \pi q}{\kappa}\left[N_{c} F_{1 / 2}\left(\frac{F_{L}+q \varphi}{k_{B} T}\right)-n\right]
$$

with

$$
n=N_{c} F_{1 / 2}\left(\frac{F_{L}}{k_{B} T}\right),
$$

the electron concentration in the emitter, $N_{c}$ is the effective density of states of the conduction band, $F_{1 / 2}(x)$ the FermiDirac integral of index $1 / 2,{ }^{27}$ and $\kappa$ is the static dielectric constant of the material. We note that the effect of size quantization on the electron distribution in the emitter is neglected. By integrating Eq. (11) and taking into account that $\varphi(-\infty)=\varphi^{\prime}(-\infty)=0$, we find the relation between $\varphi_{L}^{\prime}$ and $u_{L}$ on the left border of the left barrier

$$
\begin{aligned}
\varphi_{L}^{\prime}= & \sqrt{\frac{8 \pi k_{B} T}{\kappa}} \\
& \times\left[N_{c} F_{3 / 2}\left(\frac{F_{L}+q u_{L}}{k_{B} T}\right)-N_{c} F_{3 / 2}\left(\frac{F_{L}}{k_{B} T}\right)-\frac{q}{k_{B} T} n u_{L}\right]^{1 / 2} .
\end{aligned}
$$

Here $F_{3 / 2}$ is the Fermi-Dirac integral of index $3 / 2 .{ }^{27}$ To simplify the task, we suppose that the barriers are undoped and that the charge in the quantum well is placed at its middle plane, so that we can write

$$
u=u_{L}+\varphi_{L}^{\prime}\left(d_{L}+d_{\mathrm{QW}} / 2\right)
$$

and

$$
u_{R}=u+\left(\varphi_{L}^{\prime}-\frac{4 \pi}{\kappa} Q\right)\left(d_{R}+d_{\mathrm{QW}} / 2\right),
$$

with $d_{L}, d_{R}, d_{\mathrm{QW}}$ the widths of, respectively, the left battier, the right barrier, and the quantum well; $Q=\left(q N_{\mathrm{QW}}^{+}-Q_{\mathrm{QW}}\right)$ is the charge in the quantum well; and $N_{\mathrm{QW}}^{+}$is the number of charged donors in the quantum well. Furthermore, we suppose that the electron concentration in the collector is the same of that in the emitter, hence, $F_{R}=\left(F_{L}-q V\right)$ and the Poisson equation in the collector takes the form given by Eq. (11) with the change $F_{L} \rightarrow F_{R}$. By integrating this equation with the condition $\varphi(\infty)=V, \varphi^{\prime}(\infty)=0$, in analogy with Eq. (12) we obtain

$$
\begin{gathered}
-\frac{k}{4 \pi} \varphi_{L}^{\prime}+Q+\frac{k}{4 \pi} \sqrt{\frac{8 \pi k_{B} T}{\kappa}}\left[N_{c} F_{3 / 2}\left(\frac{F_{L}+q u_{R}-q V}{k_{B} T}\right)\right. \\
\left.-N_{c} F_{3 / 2}\left(\frac{F_{L}-q V}{k_{B} T}\right)-\frac{q n}{k_{B} T}\left(u_{R}-V\right)\right]^{1 / 2}=0 .
\end{gathered}
$$

Equation (15) relates $u_{R}$ to $V$. We remark that it is more convenient to consider $V$ and $\langle I\rangle$ as functions of $u$ because in this case they are single valued functions even for $I-V$ characteristics of $Z$ type. Since the first and the third term in the left hand side of Eq. (15) are the charge of the emitter $Q_{L}$ and of the collector $Q_{R}$, respectively, Eq. (15) expresses the electroneutrality condition of the device. We note, that to derive Eq. (15) we have assumed that the width of the depletion region in the collector region (see Fig. 1) is smaller than that 
between the right barrier and the highly doped region in the collector. As typical in DBRDs, from both sides of the barriers there are spacers with doping level of the order of $10^{16}-10^{17} \mathrm{~cm}^{-3}$ and with widths in the range between 10 and $500 \mathrm{~nm} \cdot{ }^{8,28}$ If the low doped region in the collector is fully depleted, then Eq. (13) must be substituted with

$$
V=u_{R}+\varphi_{R}^{\prime} L-\frac{2 \pi q n L^{2}}{\kappa},
$$

where $L$ is the width of the spacer in the collector, and $\varphi_{R}^{\prime}$ $=\left(\varphi_{L}^{\prime}-4 \pi Q_{Q W} / \kappa\right)$. In the derivation of Eq. (16), the voltage drop in the highly doped collector region is neglected.

\section{NOISE}

To calculate the spectral density of current fluctuations at zero frequency under fixed voltage we use the expression ${ }^{25}$

$$
S_{I}(0)=\int_{-\infty}^{\infty} d t\langle\delta I(0) \delta I(t)+\delta I(t) \delta I(0)\rangle .
$$

We anticipate that the total current fluctuation consists of two sources. The first comes from the fluctuation of population states in the contacts and the second from the fluctuation of the electron charge in the quantum well. ${ }^{22}$ This last leads to fluctuations of the voltage drop between the emitter and the quantum well, $\delta u$. Accordingly, the operator of current fluctuations is given by ${ }^{22}$

$$
\delta I(t)=\delta I_{2}(t)+\frac{\partial\langle I\rangle}{\partial u} \delta u(t),
$$

where $\delta I_{2}(t)$ is the current fluctuation operator under fixed $u$ due to the population fluctuations in the contacts. Analogously we can introduce the operator of charge fluctuation

$$
\delta Q_{\mathrm{QW}}(t)=\delta Q_{\mathrm{QW} 2}(t)-C_{\mathrm{QW}} \delta u(t),
$$

where $C_{\mathrm{QW}}$ is the differential capacitance of the quantum well

$$
C_{\mathrm{QW}}=-\frac{\partial\left\langle Q_{\mathrm{QW}}\right\rangle}{\partial u} .
$$

From the condition of charge neutrality, the charge fluctuations in the emitter $\delta Q_{e}$, in the collector $\delta Q_{c}$, and in the quantum well $\delta Q_{\mathrm{QW}}$ satisfy

$$
\delta Q_{e}+\delta Q_{c}+\delta Q_{\mathrm{QW}}=0 .
$$

The charge fluctuation in the emitter and collector can be expressed through $\delta u_{L, R}$ as

$$
\delta Q_{e}=-C_{e} \delta u_{L}, \delta Q_{c}=-C_{c} \delta u_{R},
$$

where $C_{e, c}=-\partial Q_{e, c} / \partial u_{L, R}(V=$ const $)$ are, respectively, the differential capacitance of the charge in the accumulation region of the emitter and in the depletion region of the collector whose expressions are detailed in the Appendix [we note that when Eq. (16) is valid $C_{c}=\kappa /(4 \pi L)$ ]. By taking in account that $\varphi_{L}^{\prime}=-4 \pi Q_{e} / \kappa$, from Eq. (13) we find

$$
\delta u=\delta u_{L}\left(1+C_{e} / C_{1}\right), C_{1}=\frac{\kappa}{4 \pi\left(d_{L}+d_{\mathrm{QW}} / 2\right)} .
$$

Analogously, by taking into account that $\varphi_{L}^{\prime}-4 \pi Q / \kappa$ $=4 \pi Q_{c} / \kappa$, from Eq. (14) the relation between $\delta u$ and $\delta u_{R}$ is found to be

$$
\delta u=\delta u_{R}\left(1+C_{c} / C_{2}\right), C_{2}=\frac{\kappa}{4 \pi\left(d_{R}+d_{\mathrm{QW}} / 2\right)} .
$$

From Eqs. (21)-(23), Eq. (20) is rewritten in the form

$$
\delta Q_{\mathrm{QW}}=\delta u\left(C_{L}+C_{R}\right), C_{L, R}=\frac{C_{e, c} C_{1,2}}{\left(C_{e, c}+C_{1,2}\right)},
$$

where $C_{L},\left(C_{R}\right)$ is the capacitance between the emitter (collector) and the center of the quantum well, respectively. From Eq. (24) it is clear that $C_{L, R}$ can be considered as two capacitances in series $C_{1,2}$ and $C_{e, c}$. If we neglect the accumulation and depletion regions (i.e., $C_{e, c} \rightarrow \infty$ ) and neglect the quantum well width (i.e., $d_{\mathrm{QW}} \rightarrow 0$ ) then $C_{L, R}$ coincide with those used in Ref. 22. From Eqs. (19) and (24), the relation between $\delta u$ and $\delta Q_{\mathrm{QW} 2}$ is found to be

$$
\delta u(t)=\frac{\delta Q_{\mathrm{QW} 2}(t)}{C_{L}+C_{R}+C_{\mathrm{QW}}} .
$$

From Eqs. (17), (18), and (25), $S_{I}(0)$ is found to be given by the sum of three terms as

$$
S_{I}(0)=S_{1}+S_{2}+S_{3} .
$$

The first, $S_{1}$, is the correlator of $\delta I_{2}$; the second, $S_{2}$, is proportional to the cross correlator between $\delta I_{2}$ and $\delta Q_{\mathrm{QW} 2}$; the third, $S_{3}$, is proportional to the correlator of $\delta Q_{\mathrm{QW} 2}$. They are given explicitely by

$$
S_{1}=\int_{-\infty}^{\infty} d t\left\langle\delta I_{2}(0) \delta I_{2}(t)+\delta I_{2}(t) \delta I_{2}(0)\right\rangle,
$$

$$
\begin{aligned}
& S_{2}= J \int_{-\infty}^{\infty} d t\left\langle\delta I_{2}(0) \delta Q_{\mathrm{QW} 2}(t)+\delta I_{2}(t) \delta Q_{\mathrm{QW} 2}(0)\right. \\
&\left.+\delta Q_{\mathrm{QW} 2}(0) \delta I_{2}(t)+\delta Q_{\mathrm{QW} 2}(t) \delta I_{2}(0)\right\rangle \\
& S_{3}=J^{2} \int_{-\infty}^{\infty} d t\left\langle\delta Q_{\mathrm{QW} 2}(0) \delta Q_{\mathrm{QW} 2}(t)+\delta Q_{\mathrm{QW} 2}(t) \delta Q_{\mathrm{QW} 2}(0)\right\rangle,
\end{aligned}
$$

where

$$
J=\frac{1}{C_{L}+C_{R}+C_{\mathrm{QW}}} \frac{\partial\langle I\rangle}{\partial u}
$$

plays the role of a differential resistance-capacitance rate.

By using the definitions for $\delta I_{\alpha 2}(t)$ : 


$$
\begin{aligned}
\delta I_{\alpha 2}(t)= & \frac{q}{2 \pi \hbar} \sum_{p_{\perp}} \sum_{\beta, p_{\beta}} \sum_{\gamma, p_{\gamma}} \exp \left[i\left(E_{\beta}-E_{\gamma}\right) t / \hbar\right] A_{\beta \gamma}^{\alpha}\left(E_{\beta}, E_{\gamma}\right) \\
& \times\left[c_{\beta}^{+}\left(p_{\beta}, p_{\perp}\right) c_{\gamma}\left(p_{\gamma}, p_{\perp}\right)-\left\langle c_{\beta}^{+}\left(p_{\beta}, p_{\perp}\right) c_{\gamma}\left(p_{\gamma}, p_{\perp}\right)\right\rangle\right]
\end{aligned}
$$

for $\delta Q_{\mathrm{QW} 2}(t)$ :

$$
\begin{aligned}
\delta Q_{\mathrm{QW} 2}(t)= & -q \sum_{p_{\perp}} \sum_{\alpha, p_{\alpha}} \sum_{\beta, p_{\beta}}\left[c_{\alpha}^{+}\left(p_{\alpha}, p_{\perp}\right) c_{\beta}\left(p_{\beta}, p_{\perp}\right)\right. \\
& \left.-\left\langle c_{\alpha}^{+}\left(p_{\alpha}, p_{\perp}\right) c_{\beta}\left(p_{\beta}, p_{\perp}\right)\right\rangle\right] \\
& \times \frac{T_{\alpha}^{*} T_{\beta} \exp \left[i\left(E_{\alpha}-E_{\beta}\right) t / \hbar\right]}{\left(E_{\alpha}-E_{r}-i \Gamma / 2\right)\left(E_{\beta}-E_{r}+i \Gamma / 2\right)}
\end{aligned}
$$

and the property 22

$$
\begin{aligned}
\left\langle c_{\alpha}^{+}\left(p_{\alpha}, p_{\perp}\right) c_{\beta}\left(p_{\beta}, p_{\perp}\right) c_{\gamma}^{+}\left(p_{\gamma}, p_{\perp}^{\prime}\right) c_{\delta}\left(p_{\delta}, p_{\perp}^{\prime}\right)\right\rangle & -\left\langle c_{\alpha}^{+}\left(p_{\alpha}, p_{\perp}\right) c_{\beta}\left(p_{\beta}, p_{\perp}\right)\right\rangle\left\langle c_{\gamma}^{+}\left(p_{\gamma}, p_{\perp}^{\prime}\right) c_{\delta}\left(p_{\delta}, p_{\perp}^{\prime}\right)\right\rangle \\
= & \delta_{\alpha \delta} \delta_{p_{\alpha} p_{\delta}} \delta_{\beta \gamma} \delta_{p_{\beta} p_{\gamma}} \delta_{p_{\perp} p_{\perp}^{\prime}} f_{\alpha}\left(E_{\alpha}+p_{\perp}^{2} / 2 m, F_{\alpha}\right) \\
& \times\left[1-f_{\beta}\left(E_{\beta}+p_{\perp}^{2} / 2 m, F_{\beta}\right)\right]
\end{aligned}
$$

it is possible to find for $S_{i} i=1,2,3$ the expressions

$$
\begin{gathered}
S_{1}=\frac{q^{2} m}{\pi^{2} \hbar^{3}} \int_{-g_{L}}^{\infty} d \varepsilon_{z} \int_{0}^{\infty} d \varepsilon_{\perp}\left\{D\left[f_{L}\left(1-f_{R}\right)++f_{R}\left(1-f_{L}\right)\right]\right. \\
\left.-D^{2}\left(f_{L}-f_{R}\right)^{2}\right\}, \\
S_{2}=-\lambda \frac{q^{2} m}{\pi^{2} \hbar^{3}} \int_{-g_{L}}^{\infty} d \varepsilon_{z} \int_{0}^{\infty} d \varepsilon_{\perp} D^{2}\left\{\frac{2 \Gamma_{L}}{\Gamma} f_{L}\left(1-f_{L}\right)\right. \\
\left.-\frac{2 \Gamma_{R}}{\Gamma} f_{R}\left(1-f_{R}\right)+\frac{\left(\Gamma_{R}-\Gamma_{L}\right)}{\Gamma}\left[f_{L}\left(1-f_{R}\right)+f_{R}\left(1-f_{L}\right)\right]\right\}, \\
S_{3}=\lambda^{2} \frac{q^{2} m}{\pi^{2} \hbar^{3}} \int_{-g_{L}}^{\infty} d \varepsilon_{z} \int_{0}^{\infty} d \varepsilon_{\perp} D^{2}\left\{\frac{\Gamma_{L}^{2}}{\Gamma^{2}} f_{L}\left(1-f_{L}\right)\right. \\
\left.+\frac{\Gamma_{L} \Gamma_{R}}{\Gamma^{2}}\left[f_{L}\left(1-f_{R}\right)+f_{R}\left(1-f_{L}\right)\right]\right\} \\
\quad \times \lambda^{2} \frac{q^{2} m}{\pi^{2} \hbar^{3}} \int_{-q V}^{\infty} d \varepsilon_{z} \int_{0}^{\infty} d \varepsilon_{\perp} D^{2} \frac{\Gamma_{R}^{2}}{\Gamma^{2}} f_{R}\left(1-f_{R}\right) .
\end{gathered}
$$

Here we used the notation $f_{L, R}=f_{L, R}\left(\varepsilon, F_{L, R}\right)$ and

$$
\lambda=\frac{\hbar \Gamma}{\Gamma_{L} \Gamma_{R}} \frac{1}{\left(C_{L}+C_{R}+C_{\mathrm{QW}}\right)} \frac{\partial\langle I\rangle}{\partial u},
$$

where $-\infty<\lambda<\infty$ is a dimensionless parameter describing Coulomb interaction.

Equations (32)-(34) are the central result of this paper. We note that $S_{I}(0)$ does not depend on $\eta$. When the $\lambda=0$, i.e.,
Coulomb interaction is negligible, $S_{I}(0)=S_{1}$ and the result of Ref. 29 is recovered.

As an internal check we prove that $S_{I}(0)$ satisfies the Nyquist theorem. ${ }^{30}$ Indeed, at zero applied voltage $f_{L}=f_{R}$, from the expression (8) for the total current it follows that $\partial\langle I\rangle / \partial u=0$, and therefore, $\lambda=0$. Accordingly, the differential conductance $G$ at zero voltage is

$$
G=\frac{q^{2} m}{2 \pi \hbar^{3} k_{B} T} \int_{0}^{\infty} d \varepsilon_{z} D \int_{0}^{\infty} d \varepsilon_{\perp} f_{L}\left(1-f_{L}\right) .
$$

We here used the property that for $V=0 \partial f_{R} / \partial q V=f_{L}(1$ $\left.-f_{L}\right) /\left(k_{B} T\right)$. According to Eq. (32), $S_{I}$ at zero voltage is

$$
S_{I}(0)=\frac{2 q^{2} m}{\pi \hbar^{3}} \int_{0}^{\infty} d \varepsilon_{z} D \int_{0}^{\infty} d \varepsilon_{\perp} f_{L}\left(1-f_{L}\right) .
$$

Equations (35) and (36) imply $S_{I}(0)=4 k_{B} T G$, which represents the Nyquist theorem.

Let us now show that $S_{I}(0) \rightarrow \infty$ on the border of the instability region where $d\langle I\rangle / d V \rightarrow \infty$. To this purpose, we note that $d I / d V$ can be decomposed as

$$
\frac{d\langle I\rangle}{d V}=\frac{\partial\langle I\rangle}{\partial V}+\frac{\partial\langle I\rangle}{\partial u} \frac{d u}{d V} .
$$

By writing the condition of charge neutrality as

$$
-C_{e} d u_{L}+d Q_{\mathrm{QW}}+C_{c}\left(d V-d u_{R}\right)=0
$$

by taking into account Eqs. (22) and (23) and that

$$
d Q_{\mathrm{QW}}=-C_{\mathrm{QW}} d u+\frac{\partial N_{\mathrm{QW}}}{\partial V} d V,
$$

we obtain

$$
\frac{d u}{d V}=\left(C_{c}+\frac{\partial Q_{\mathrm{QW}}}{\partial V}\right)\left(C_{L}+C_{R}+C_{\mathrm{QW}}\right)^{-1} .
$$

By using Eq. (8) for $\langle I\rangle$, we see that $\partial\langle I\rangle / \partial u_{L}$ and $\partial\langle I\rangle / \partial V$ entering Eq. (37) are always finite. This implies that $d\langle I\rangle / d V \rightarrow \infty$ only if the sum $\left(C_{L}+C_{R}+C_{\mathrm{QW}}\right) \rightarrow 0$. We remark that also the denominator of $\lambda$ is proportional to $\left(C_{L}+C_{R}\right.$ $\left.+C_{\mathrm{QW}}\right)$; thus, $\lambda$, and in turn $S_{I}(0)$ go to infinity simultaneously with $d I / d V$. Note that $C_{L, R}$ are always positive and $C_{\mathrm{QW}}$ becomes negative when the resonant state approaches $g_{L}$, that is when the number of electrons in the quantum well decreases at increasing $u$.

\section{RESULTS AND DISCUSSION}

In this section we present the theoretical results for the two relevant cases of low temperatures (i.e., $T \leqslant 4.2 \mathrm{~K}$ ) and high temperatures (i.e., $T \gg \Gamma$ which here corresponds to $T$ $\geqslant 77 \mathrm{~K}$ ), the former being appropriate to investigate the influence on shot noise of the Pauli principle and the latter of long range Coulomb interaction. Then, theoretical results are compared with experiments. 


\section{A. Low temperature}

We investigate the condition of high applied voltages, when $q V>F_{R}$, because more close to experiments. (We recall that typical magnitudes for the relevant parameters of DBRDs are: for $\Gamma$ less than a few milli-electron-volts, for $F_{L}$ less than $100 \mathrm{meV}$, and for the voltage at the peak current around 0.5 V.) Because of the above, from Eq. (8) the expression for the current becomes

$$
\langle I\rangle=\frac{q m}{2 \pi^{2} \hbar^{3}} \int_{-g_{L}}^{F_{L}} d \varepsilon\left(F_{L}-\varepsilon\right) D(\varepsilon)=\frac{q m \Gamma_{L} \Gamma_{R}}{4 \pi^{2} \hbar^{2}} B(f, \xi),
$$

where

$$
\begin{aligned}
B(f, \xi)= & 2(f+\xi)[\arctan (f+\xi)-\arctan (\xi)] \\
& -\ln \left[\frac{1+(f+\xi)^{2}}{1+\xi^{2}}\right],
\end{aligned}
$$

and for convenience we define dimensionless chemical potential $f$ and voltage drop $\xi$ as

$$
f=\frac{2\left(F_{L}+g_{L}\right)}{\Gamma}, \xi=\frac{2\left(q u-\varepsilon_{r}-g_{L}\right)}{\Gamma} .
$$

The expression for the noise spectral density becomes

$$
\begin{aligned}
S_{I}(0)= & \frac{q^{2} m}{\pi^{2} \hbar^{3}} \int_{-g_{L}}^{F_{L}} d \varepsilon\left(F_{L}-\varepsilon\right) D(\varepsilon)[1-D(\varepsilon)] \\
& +\frac{q^{2} m}{\pi^{2} \hbar^{3}}\left[\lambda \frac{\left(\Gamma_{L}-\Gamma_{R}\right)}{\Gamma}+\lambda^{2} \frac{\Gamma_{L} \Gamma_{R}}{\Gamma^{2}}\right] \int_{-g_{L}}^{F_{L}} d \varepsilon\left(F_{L}-\varepsilon\right) D(\varepsilon)^{2} .
\end{aligned}
$$

For the Fano factor, $\gamma=S_{I}(0) / 2 q\langle I\rangle$, Eqs. (41) and (42) yield

$$
\gamma=1-\frac{4 \Gamma_{L} \Gamma_{R}}{\Gamma^{2}}\left[1-\lambda \frac{\left(\Gamma_{L}-\Gamma_{R}\right)}{\Gamma}-\lambda^{2} \frac{\Gamma_{L} \Gamma_{R}}{\Gamma^{2}}\right] \frac{A(f, \xi)}{B(f, \xi)},
$$

where

$$
\begin{aligned}
A= & (f+\xi)\left[\frac{f+\xi}{1+(f+\xi)^{2}}+\arctan (f+\xi)-\frac{\xi}{1+\xi^{2}}-\arctan (\xi)\right] \\
& +\frac{1}{1+(f+\xi)^{2}}-\frac{1}{1+\xi^{2}},
\end{aligned}
$$

We note that $\lambda$ depends on $f$ and $\xi$ through $C_{L, R}, C_{\mathrm{QW}}$, $\partial\langle I\rangle / \partial u$ which are given by Eq. (24) and by

$$
C_{\mathrm{QW}}=\frac{q^{2} m}{\pi^{2} \hbar^{2}} \frac{\Gamma_{L}}{\Gamma} H(f, \xi), \frac{\partial\langle I\rangle}{\partial u}=\frac{q^{2} m \Gamma_{L} \Gamma_{R}}{\pi^{2} \hbar^{3} \Gamma} H(f, \xi),
$$

$$
\begin{aligned}
H(f, \xi)= & {\left[\arctan (f+\xi)-\arctan (\xi)-\frac{f}{\xi^{2}+1}\right] } \\
& +\frac{\Gamma^{2}}{4 \Gamma_{L} \Gamma_{R}} \frac{\partial g_{L}}{\partial q u} f D\left(-g_{L}\right) .
\end{aligned}
$$

If $f \gg 1$ and the resonant level is located below the the Fermi level of the emitter far from its borders $F_{L}$ and $-g_{L}$, i.e., $\xi$ $\ll-1, f+\xi \gg 1$, then $A(f, \xi) / B(f, \xi) \approx 1 / 2$ and Eq. (43) recovers the expression given in Ref. 22 :

$$
\gamma=\frac{\Gamma_{L}^{2}+\Gamma_{R}^{2}+2 \Lambda\left(\Gamma_{R}-\Gamma_{L}\right)+2 \Lambda^{2}}{\Gamma^{2}}
$$

with

$$
\Lambda=-\frac{\lambda \Gamma_{L} \Gamma_{R}}{\Gamma}
$$

As it will be shown later, when $f \gg 1$ the values taken by $\gamma$ in Eq. (45) are practically constant and correspond to the plateau exhibited by the dependence $\gamma(f, \xi)$. On this plateau, $\gamma \geqslant 1 / 2$ [the minimum is reached when $\Lambda=\left(\Gamma_{L}-\Gamma_{R}\right) / 2$ ]. Note that on the plateau

$$
C_{\mathrm{QW}} \approx \frac{q^{2} m \Gamma_{L}}{\pi \hbar^{2} \Gamma}, \lambda \approx \frac{q^{2} m}{\pi \hbar^{2}} \frac{1}{C_{L}+C_{R}+C_{\mathrm{QW}}}<\frac{\Gamma}{\Gamma_{L}}
$$

and the expression in the braces of Eq. (43) is positive. Therefore, on the plateau $1 \geqslant \gamma \geqslant 1 / 2$ and shot noise enhancement is impossible.

Now we demonstrate that at voltage values for which the resonant level is close to the band edge of the emitter the Fano factor can drop below the value $1 / 2$. To this purpose, let us first consider the simplified model where we take $\kappa$ $=12.9$ (GaAs), $C_{L}=C_{R}=\kappa / 4 \pi d, d=5 \mathrm{~nm}$ and neglect the term proportional to $\partial g_{L} / \partial u$ in the expression for $H(f, \xi)$. Figure 2 reports the dependencies of current and Fano factor on $\xi$ for the two values $f=100$ [Fig. 2(a)], $f=10$ [Fig. 2(b)] when $\Gamma_{L}=\Gamma_{R}$ and in the presence (continuous curves) or absence (dotted curves) of Coulomb interaction. The figure shows that for large value of the ratio the resonant width $(f=100), \quad \gamma(\xi)$ exhibits a wide plateau region where $\gamma$ $\approx 0.55$ followed by a minimum with $\gamma_{\text {min }} \approx 0.464$. By further increasing the value of $f(f=1000)$ the plateau region is found to widen and $\gamma_{\min } \approx 0.49$. Note that, as it follows from Eq. (42), the Coulomb interaction always increases the noise if $\Gamma_{L}=\Gamma_{R}$. With the decrease of $f$ [see Fig. 2(b) where $f=10$ ], the plateau region becomes narrower and $\gamma_{\min }$ is found to decrease. The decrease of the value of $\gamma_{\min }$ is due to two complementary reasons. The first is associated with the decreasing of the strength of Coulomb interaction. The second is associated with the increase of the effective barrier transparency near to the current peak and in turn with the further suppression of partition noise. For the ideal case $f \ll 1$, at the peak current the transparency $D \rightarrow 1$ and in turn $\gamma_{\min } \rightarrow 0$.

Why does Coulomb interaction increase the shot noise if $\Gamma_{L}=\Gamma_{R}$ ? To answer this question, we analyze the noise contribution due to electrons with energies implying $D \rightarrow 1$. The part of $S_{1}$ corresponding to these electrons is zero, while the part of $S_{3}$ is finite. We remind that the term $S_{1}$ describes 

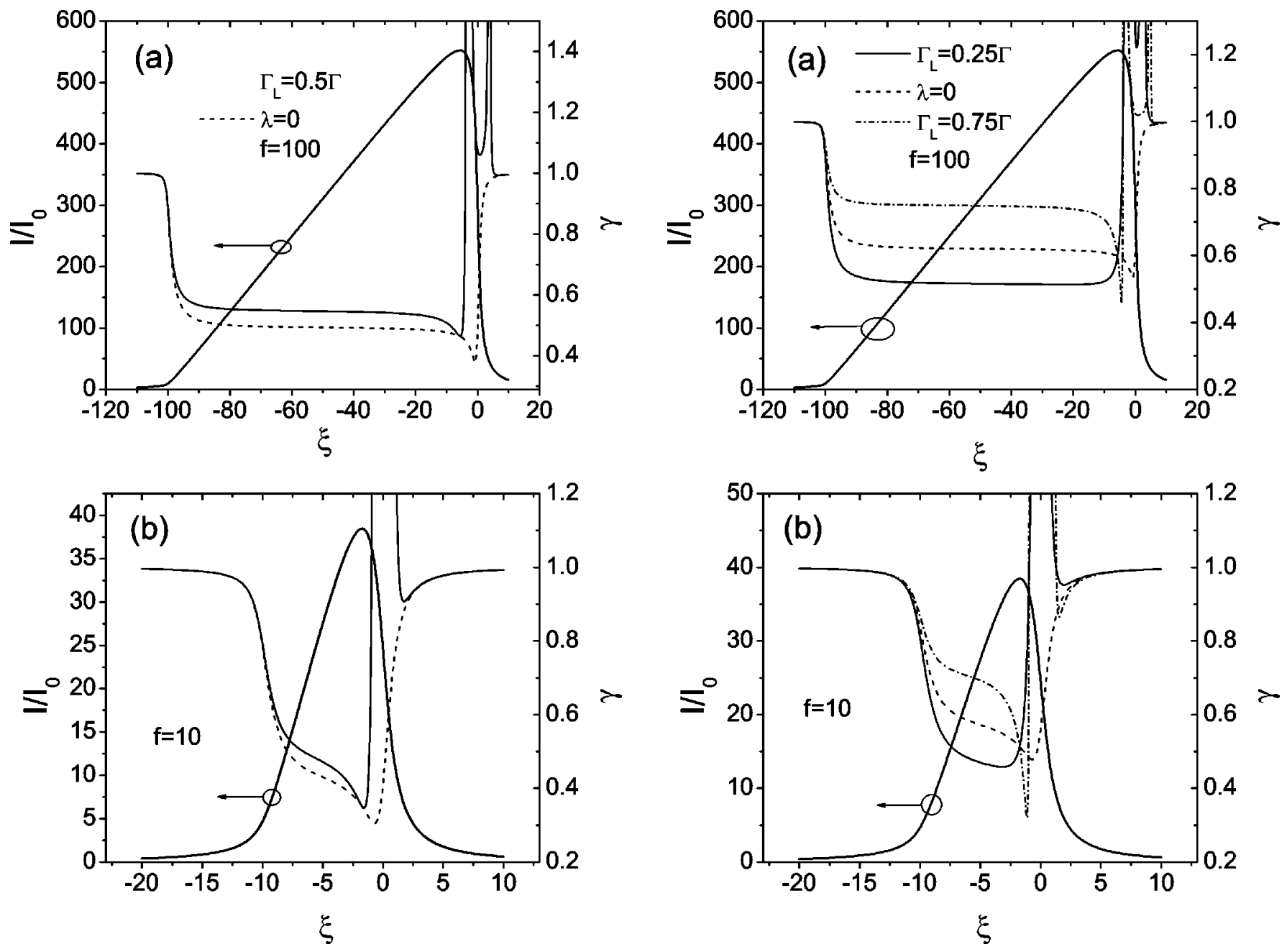

FIG. 2. Dependence of the Fano factor and of the current on the electrical potential in dimensionless units $\xi=2\left(q u-\varepsilon_{r}-g_{L}\right) / \Gamma$ with $f=100$ (a) and $f=10$ (b) for the symmetrical structure $\Gamma_{L}=\Gamma_{R}$. Here $f=2\left(F_{L}+g_{L}\right) / \Gamma$ and $I_{0}=q m \Gamma_{L} \Gamma_{R} /\left(2 \pi^{2} \hbar^{3}\right)$. Continuous (dashed) curves correspond to the presence (absence) of Coulomb interaction.

partition noise, thus it is proportional to $D(1-D)$ and for $D=1$ at zero frequency there is no noise associated with the fluctuation of $\delta I_{2}$. However, even for $D=1$ there is charge fluctuation in the quantum well due to the random character of electron escape from the well (random time delay of charge). The probability of this escape is proportional to $\Gamma$ and this is the reason why $S_{3}$ decreases with the increase of $\Gamma$. Therefore, Coulomb interaction enhances current noise due to the random time delay of the charge in the quantum well, and which represents the quantum property of the electron motion in the RTD.

The effects due to the asymmetry of the diode barriers when $\Gamma_{L}=0.25 \Gamma$ and $\Gamma_{L}=0.75 \Gamma$ are shown in Fig. 3 which reports the dependencies of current and Fano factor on $\xi$ for the two values $f=100, f=10$. We note, that without Coulomb interaction (i.e., $\lambda=0$ ) these dependencies are the same for $\Gamma_{L}=0.25 \Gamma$ and $\Gamma_{L}=0.75 \Gamma$. From this figure it is clear that on the plateau Coulomb interaction decreases the noise for $\Gamma_{L}$ $=0.25 \Gamma$ while increases the noise for $\Gamma_{L}=0.75 \Gamma$. This asym-

FIG. 3. Dependence of the Fano factor and the current on the electrical potential for asymmetric structures with $f=100$ (a) and $f=10$ (b). The dimesionless units are the same of Fig. 2.

metry is due to the cross correlation term $S_{2}$ which on the plateau is positive for $\Gamma_{L}>\Gamma_{R}$ and negative for $\Gamma_{L}<\Gamma_{R}$. The physical reason of this asymmetry stems from the following fact. If $\Gamma_{L}>\Gamma_{R}$, then the charge situated in the quantum well is characterized by an escaping probability to the emitter which is greater than that to the collector. By contrast, when $\Gamma_{L}<\Gamma_{R}$ the opposite happens. Of course, when $\Gamma_{L}=\Gamma_{R}$ the escaping probability to the emitter and to the collector is the same and $S_{2}=0$. We note the important role played by the Pauli principle for the possibility of $S_{2}$ to be positive. From Eq. (33) it is clear that if $f_{L} \ll 1$ and $f_{R}=0$ then $S_{2}<0$ for any value of the ratio $\Gamma_{L} / \Gamma_{R}$. From Fig. 3 one can also see that $\gamma_{\min }=0.457<0.5$ for $\Gamma_{L}=0.75 \Gamma$ and $f=100$ [see Fig. 3(a)] and for both values of $\Gamma_{L}$ when $f=10$ [see Fig. 3(b)].

We conclude, that for the simple model considered here both the increase of $\Gamma$ and the decrease of $F_{L}$ decreases $\gamma_{\text {min }}$ which value drops below 0.5 for $\Gamma_{L}>\Gamma_{R}$. We further note, that there is bias when $\partial\langle I\rangle / \partial u=0$ and $\lambda$ changes of sign. Under this bias, for $\Gamma_{L}=\Gamma_{R}$ the curves of the Fano factor with and without Coulomb interaction touch each other (see Fig. 2) while for $\Gamma_{L} \neq \Gamma_{R}$ they cross (see Fig. 3).

To confirm the possibility of evidencing the giant suppression $\gamma_{\min }<0.5$ in a real structure, Fig. 4 presents the calcu- 


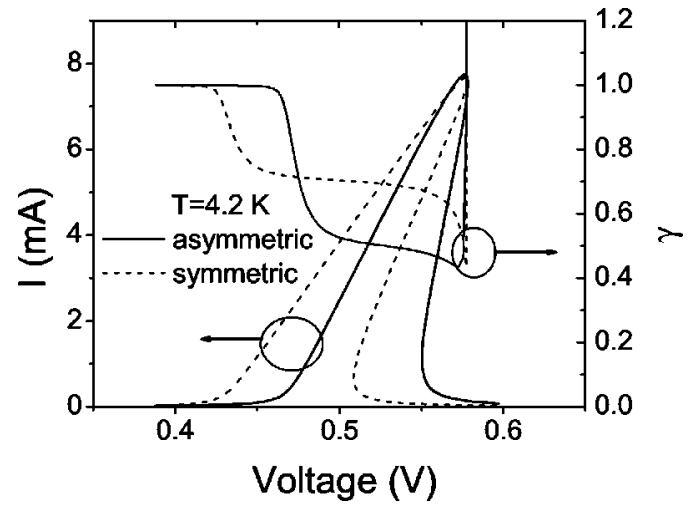

FIG. 4. Dependence of the current and Fano factor on the applied voltage for the structure of Ref. 8 at $T=4.2 \mathrm{~K}$. Values of $\Gamma_{L, R}$ and $\varepsilon_{r}$ are chosen from the fitting of the current volatage characteristic at $77 \mathrm{~K}$.

lations performed with a set of parameters related to the experiments in Ref. 8 at $T=4.2 \mathrm{~K}$ and where both a symmetric (continuous curve) and an asymmetric (dashed curve) are considered. The structure parameters are $n=2 \times 10^{16} \mathrm{~cm}^{-3}$, $d_{L}=d_{R}=d_{\mathrm{QW}}=5 \mathrm{~nm}$, squared area of contacts $50 \mu \mathrm{m}^{2}$ and $L=50 \mathrm{~nm}, m=0.067 m_{0}$, with $m_{0}$ the free electron mass and $\kappa=12.9$. In the symmetric structure, the only two fitting parameters are $\Gamma_{L}=0.5 \Gamma=0.48 \mathrm{meV}$ and $\varepsilon_{r}=104 \mathrm{meV}$. Their values control the location and the amplitude of the current peak, respectively, and are chosen by optimizing the agreement between the experimental and calculated $I$ - $V$ characteristics at $77 \mathrm{~K}$. For the asymmetric structure there are three parameters and we take $\Gamma_{L}=0.25 \Gamma, \Gamma=1.22 \mathrm{meV}, \varepsilon_{r}$ $=112 \mathrm{meV}$ to preserve the location of the current peak at the same voltage. The function $g_{L}\left(u_{L}\right)$ is calculated by solving the Schrödinger equation for a potential which: (i) for $x<0$ follows from the Poisson equation without accounting for quantization effects; (ii) for $x>0$ corresponds to the solid solution $\mathrm{Al}_{0.42} \mathrm{Ga}_{0.58}$ As with zero electric field inside, as was done in Ref. 8. For these values, the dependence $g_{L}\left(u_{L}\right)$ is found to be almost linear and well approximated by: $g_{L}\left(u_{L}\right)$ $\approx 0.28\left(q u_{L}-110 k_{B} T\right)-14 k_{B} T$. The details of the solution of the Schrödinger equation are reported in the Appendix. Calculations give $\gamma_{\min }=0.43$ in reasonable agreement with the value of 0.35 found in experiments. ${ }^{8}$ From Fig. 4 we see that both the $I-V$ and the noise characteristics are sensitive to the asymmetry of the structure, as expected. In any case, the main features of both characteristics are preserved.

We conclude, that the main reason for shot noise suppression in RTDs at temperatures below about $4.2 \mathrm{~K}$ is essentially related to the Pauli principle and, because of the coherent tunneling regime, near to the current peak the Fano factor can take values significantly lower than 0.5 (giant shot-noise suppression).

\section{B. High temperature}

We now discuss the case of high temperatures when $k_{B} T$ $\gg \Gamma$, which in the present case refers to $T \geqslant 77 \mathrm{~K}$. Again, we consider applied voltages high enough to neglect the contribution to the current and noise of the electron flux moving from the collector to the emitter. Since the energy scale for the change of $D(\varepsilon)$ is significantly less than that of $f_{L}(\varepsilon)$, all the integrals in the expressions for the current and the noise spectral density can be calculated analytically. To perform the calculations, first of all we note that

$$
\begin{aligned}
& \int_{0}^{\infty} d \varepsilon_{\perp} f_{L}(\varepsilon)=k_{B} T \ln \left[1+\exp \left(\frac{F_{L}-\varepsilon_{z}}{k_{B} T}\right)\right]=k_{B} T \Phi_{L}\left(\varepsilon_{z}\right) \\
& \begin{aligned}
\int_{0}^{\infty} d \varepsilon_{\perp} f_{L}(\varepsilon)^{2} & =k_{B} T\left\{\Phi_{L}\left(\varepsilon_{z}\right)-\left[1+\exp \left(\frac{F_{L}-\varepsilon_{z}}{k_{B} T}\right)\right]^{-1}\right\} \\
& =k_{B} T \Phi_{2 L}\left(\varepsilon_{z}\right)
\end{aligned}
\end{aligned}
$$

and

$$
\begin{aligned}
& \int_{-g_{L}}^{\infty} d \varepsilon_{z} D\left(\varepsilon_{z}\right) \Phi_{L, 2 L}\left(\varepsilon_{z}\right) \approx \frac{2 \Gamma_{L} \Gamma_{R}}{\Gamma} \Phi_{L, 2 L}(\beta) D_{1}(\xi), \\
& \int_{-g_{L}}^{\infty} d \varepsilon_{z} D\left(\varepsilon_{z}\right)^{2} \Phi_{L, 2 L}\left(\varepsilon_{z}\right) \approx \frac{4 \Gamma_{L}^{2} \Gamma_{R}^{2}}{\Gamma^{3}} \Phi_{L, 2 L}(\beta) D_{2}(\xi),
\end{aligned}
$$

here $\beta$ is the maximum value between $\left(\varepsilon_{r}-q u\right)$ and $-g_{L}$ :

$$
D_{1}(\xi)=\frac{\pi}{2}-\arctan (\xi), D_{2}(\xi)=D_{1}-\frac{\xi}{\xi^{2}+1}
$$

By using Eqs. (46)-(49), we can write the following expressions, respectively for the current, the noise spectral density and the Fano factor

$$
\begin{gathered}
\langle I\rangle=\frac{q m k_{B} T \Gamma_{L} \Gamma_{R}}{\pi^{2} \hbar^{3} \Gamma} \Phi_{L}(\beta) D_{1}(\xi) \\
S_{I}(0)=\frac{2 q^{2} m k_{B} T \Gamma_{L} \Gamma_{R}}{\pi^{2} \hbar^{3} \Gamma}\left(D_{1}(\xi) \Phi(\beta)\right. \\
-\frac{2 \Gamma_{L} \Gamma_{R} D_{2}(\xi)}{\Gamma^{2}}\left\{\Phi_{2 L}(\beta)+\lambda\left[\Phi_{L}(\beta)-\frac{2 \Gamma_{L}}{\Gamma} \Phi_{2 L}(\beta)\right]\right. \\
\left.\left.-\lambda^{2} \frac{\Gamma_{L}}{\Gamma}\left[\Phi_{L}(\xi)-\frac{\Gamma_{L}}{\Gamma} \Phi_{2 L}(\beta)\right]\right\}\right), \\
\gamma=1-\frac{2 \Gamma_{L} \Gamma_{R} D_{2}(\xi)}{\Gamma^{2} D_{1}(\xi)}\left\{\Phi(\beta)+\lambda\left[1-\frac{2 \Gamma_{L}}{\Gamma} \Phi(\beta)\right]\right. \\
\left.-\lambda^{2} \frac{\Gamma_{L}}{\Gamma}\left[1-\frac{\Gamma_{L}}{\Gamma} \Phi(\beta)\right]\right\},
\end{gathered}
$$

here $\Phi(\beta)=\Phi_{2 L}(\beta) / \Phi_{L}(\beta)$. Analogously, we derive the expressions for $C_{\mathrm{QW}}$ and $\partial<I>/ \partial u$. Since in this case $\partial<I>/ \partial u=\Gamma_{R} C_{\mathrm{QW}} / \hbar$, we obtain 


$$
\lambda=\frac{\Gamma C_{\mathrm{QW}}}{\Gamma_{L}\left(C_{L}+C_{R}+C_{\mathrm{QW}}\right)}
$$

and $\lambda<\Gamma / \Gamma_{L}$ in the stable region where $\left(C_{L}+C_{R}\right.$ $\left.+C_{\mathrm{QW}}\right)>0$.

We now estimate the possible minimum value for $\gamma$. From Eq. (52) it is clear that to obtain $\gamma_{\min }$ it is necessary that

$$
\lambda=\frac{\Gamma}{2 \Gamma_{L}}\left[1-\frac{2 \Gamma_{L}}{\Gamma} \Phi(\beta)\right]\left[1-\frac{\Gamma_{L}}{\Gamma} \Phi(\beta)\right]^{-1} .
$$

We note, that this value of $\lambda$ lies in the range of possible values characterizing the stable region. By substituting Eq. (54) into Eq. (52) we find

$$
\gamma_{\min }=1-\frac{\Gamma_{R}}{2 \Gamma} \frac{D_{2}(\xi)}{D_{1}(\xi)}\left[1-\frac{\Gamma_{L}}{\Gamma} \Phi(\beta)\right]^{-1} .
$$

Now we analyze two limiting cases.

The first is the case when the occupation factor of the state with energy $\beta$ is much smaller than unity. Then $\Phi(\beta)$ $\approx 0$ and

$$
\gamma_{\min }=1-\frac{\Gamma_{R}}{2 \Gamma} \frac{D_{2}(\xi)}{D_{1}(\xi)} .
$$

Since the maximum value of $D_{2}(\xi) / D_{1}(\xi)$ equals 1.217 ( $\xi$ $=\xi_{0}=-0.802$ ), from Eq. (56) we find that $\gamma_{\min }<0.5$ when $\Gamma_{R}>0.821 \Gamma$, and that the expression (54) holds near $\xi=\xi_{0}$.

The second limiting case is when the probability of occupation of an electron state with energy $\beta$ is close to unity and $\Phi(\beta) \approx 1$. In this case

$$
\gamma_{\min }=1-\frac{D_{2}(\xi)}{2 D_{1}(\xi)}
$$

and $\gamma_{\min }<0.5$ when the expression (54) holds in any point where $\xi<0$, since there $D_{2}(\xi) / D_{1}(\xi)>1$.

In concluding this subsection, we emphasize that, for RTD theory predicts values of the Fano factor below 0.5 also at high temperatures.

\section{Comparison of theory with experiments}

We compare theory with two sets of experiments performed on DBRDs with barriers sufficiently narrow to expect that coherent tunneling is of importance. The first set refers to pioneer experiments of Brown ${ }^{8}$ which are detailed at $77 \mathrm{~K}$ with indications at $300 \mathrm{~K}$. The second set refers to recent experiments at $300 \mathrm{~K}$ reported in Refs. 31 and 32 . In both cases the comparison is limited to the voltage region up to the peak current since theory neglects energy levels in the quantum well higher than the first one.

Figure 5 reports the comparison between experiments performed in Ref. 8 and present calculations at $77 \mathrm{~K}$. Numerical results make use of the same parameters in Fig. 4 for the symmetric structure. For the used values, the dependence $g_{L}\left(u_{L}\right)$ is found to be almost linear and well approximated by: $g_{L}\left(u_{L}\right) \approx 0.44\left(q u_{L}-1.5 k_{B} T\right)-0.07 k_{B} T$ for $77 \mathrm{~K}$.

From Fig. 5(a) we see that present calculations well reproduce the $I-V$ characteristic including the current peak.
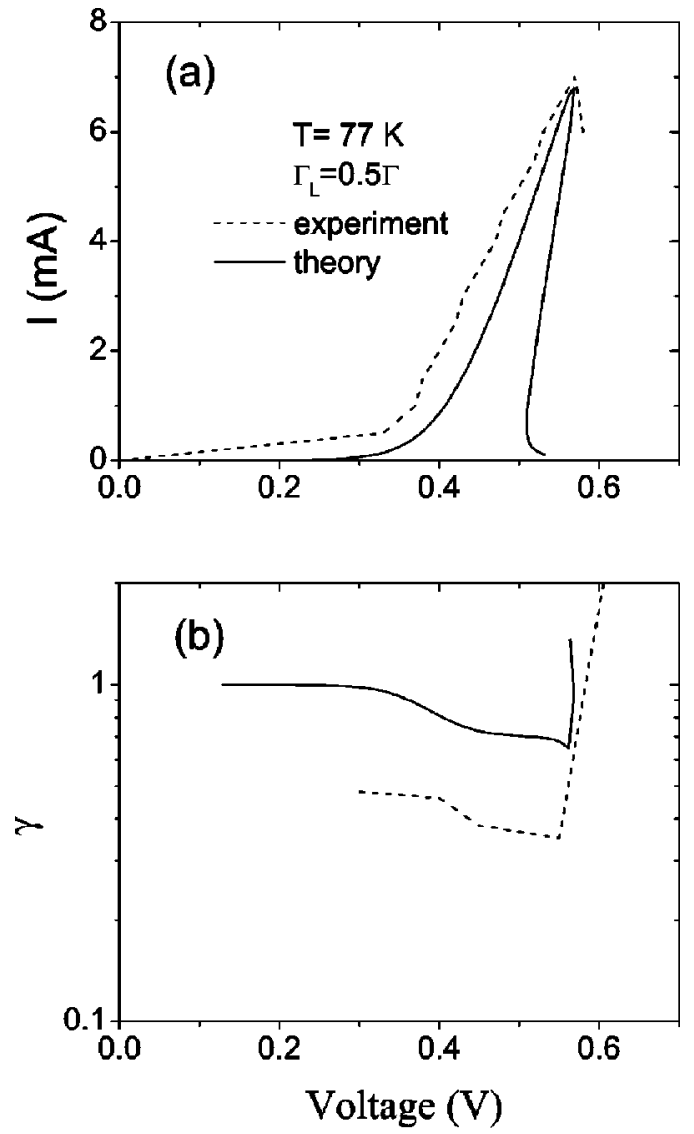

FIG. 5. Calculated (solid) and measured (dashed) dependencies of current and Fano factor on the applied voltage for the structure of 8 and $77 \mathrm{~K}$. The parameters are the same used for Fig. 4 in the case of the symmetric structure.

From Fig. 5(b) we see that the calculated Fano factor reproduces both the suppression and enhanced behaviors. However, its minimum value is of 0.65 against the experimental value of 0.35 . By choosing appropriate values of $\Gamma_{L, R}$ the theoretical model can be forced to fit the experimental Fano factor at the expense of overestimating the $I-V$ characteristics for about one order of magnitude. This result corrects previous findings of the same authors, ${ }^{32}$ where the contribution of charge fluctuations to the total noise was underestimated with respect to the present approach. In this context, we note that Ref. 16 presented a theoretical calculations of the same experiments $^{8}$ at $77 \mathrm{~K}$. The results of these calculations were found to be in excellent agreement with experimental data except for the region of instability. On the borders of this region the noise tends to infinity and, as a consequence, there were no measurements of noise in this region. However, contrary to such an experimental evidence, the theoretical calculations ${ }^{16}$ provided finite values of the noise and the the absence of the instability region, which makes the theoretical approach at least suspect.

To provide a physical insight of the physical reason for shot-noise suppression, Fig. 6 reports the results of the calculations associated with the presence (continuous curves) and the absence (dotted lines) of Coulomb interaction. From Fig. 6(a) we see that at $77 \mathrm{~K}$ the Coulomb part contributes to suppress shot noise in the whole region of applied voltage, 

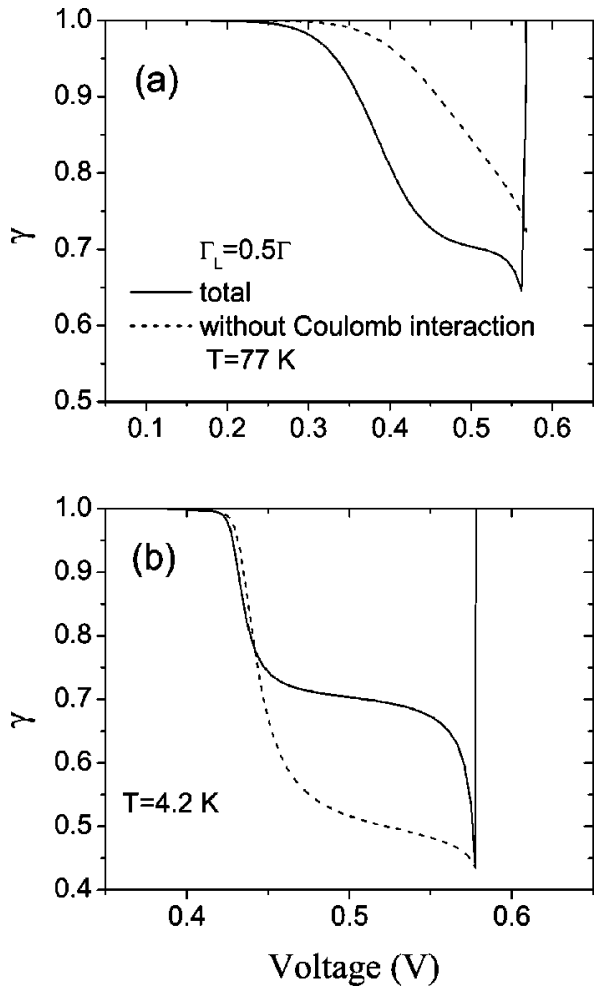

FIG. 6. Calculated dependencies of the Fano factor with (continuous curves) and without (dashed curves) Coulomb interaction at 4.2 and 77 K. Other parameters are as in Fig. 5.

and that the Pauli part becomes noticeable near the current peak. Furthermore, while the Pauli contribution leads systematically to suppression, the Coulomb contribution becomes responsible of enhanced shot noise at voltages near to $0.57 \mathrm{~V}$ where the instability region is approached. From Fig. 6(b) we see that at $4.2 \mathrm{~K}$ the predominance of Pauli over Coulomb interaction in suppressing shot noise is confirmed. Again, Coulomb effects are found to be responsible of enhanced shot noise around above the current peak, in agreement with experiments. ${ }^{8}$

Figure 7 reports the current voltage characteristic and the Fano factor for the same structure of Fig. 5 at $300 \mathrm{~K}$. Here the solution of the Schrödinger equation provides: $g_{L}\left(u_{L}\right)$ $=0.53\left(q u_{L}-1.5 k_{B} T\right)+0.7 k_{B} T$. Calculations show, that even by increasing the temperature, the main features of transport and noise already shown at $77 \mathrm{~K}$ are preserved. However, the current peak and the minimum of the Fano factor become less pronounced at increasing the temperature. These trends are in agreement with experimental results ${ }^{8}$ which claim a reduction of the peak-current value of about $1 \mathrm{~mA}$ and an increase of the minimum of the Fano factor when going from 77 to $300 \mathrm{~K}$. However, even at $300 \mathrm{~K}$ present calculations give a Fano Factor for about a factor of 2 greater than that found by experiments.

A recent set of experiments ${ }^{31,32}$ carried out at $300 \mathrm{~K}$ on a DBRD with barriers thinner than those of Ref. 8, thus more adequate to check coherent tunneling at high temperature, is reported in Fig. 8 together with theoretical calculations. The structure consisted of two $2 \mathrm{~nm}$ AlAs layers separated by $6 \mathrm{~nm}$ InGaAs quantum well. ${ }^{33,31}$ Measurements were carried
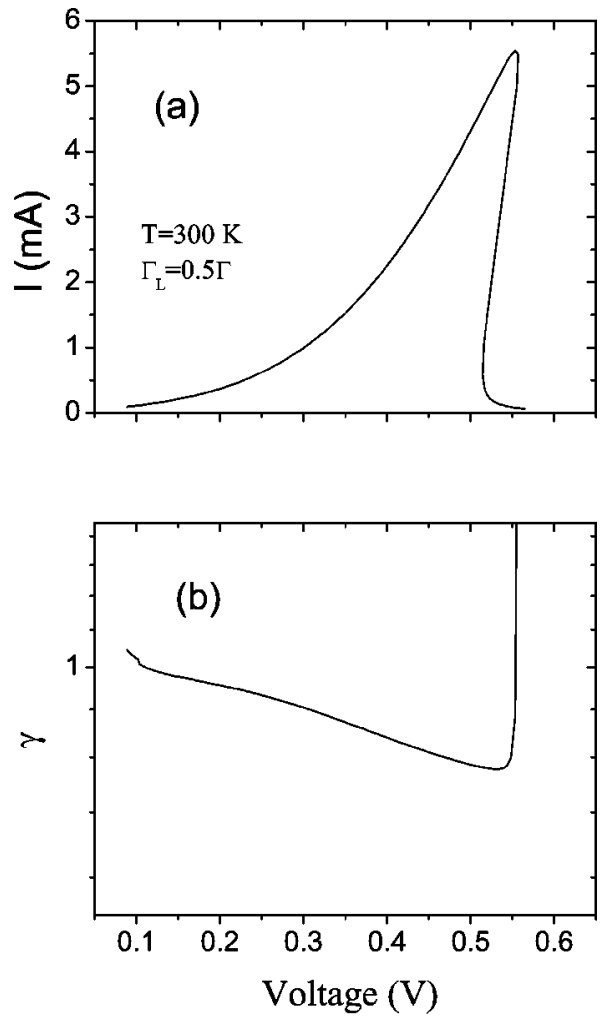

FIG. 7. Dependencies of current and Fano factor on applied voltage at $T=300 \mathrm{~K}$. Other parameters are as in Fig. 5.

out at $300 \mathrm{~K}$ using a noise figure meter (XK5-49), that allows to measure simultaneously noise figure and power gain of two-port networks in the $50 \Omega$ feed circuit. Simultaneously with the noise the $I-V$ curve was measured. The diode was mounted in the break of a microstrip line, with one electrode been grounded, and another one bonded to the ends of a microstrip line. Noise measurements at frequencies 60 and $200 \mathrm{MHz}$ showed the same results within an experimental uncertainty at worst of $20 \%$, thus indicating that $1 / f$ noise contribution is negligible. Numerical results makes use of the following values for the parameters entering the model: $\varepsilon_{r}=87 \mathrm{meV}, \Gamma_{R}=\Gamma_{L}=1.08 \mathrm{meV}, n=5 \times 10^{16} \mathrm{~cm}^{-3}$, $d_{L}=d_{R}=2 \mathrm{~nm}, \quad d_{\mathrm{QW}}=6 \mathrm{~nm}, \quad L=50 \mathrm{~nm}, \quad g_{L}\left(u_{L}\right)=0.445\left(q u_{L}\right.$ $\left.-1.5 k_{B} T\right)+0.451 k_{B} T, m=0.045 m_{0}$. Also here the only two fitting parameters are $\varepsilon_{r}$ and $\Gamma=0.5 \Gamma_{L, R}$, all other parameters being provided by the experimental conditions, Fig. 8(a) reports the $I-V$ characteristic which shows a region of positive differential conductance (pdc) up to about $0.7 \mathrm{~V}$ followed by an instability region. In the same pdc region, the Fano factor is found to exhibit a suppression with a minimum value of about 0.4 at around $0.65 \mathrm{~V}$ [see Fig. 8(b)]. As for the case at $77 \mathrm{~K}$, from Fig. 8(a) we see that present calculations well reproduce the $I-V$ characteristic including the current peak. From Fig. 8(b) we see that the calculated Fano factor reproduces both the suppression and enhanced behaviors. However, the minimum value of the Fano factor is found to be of 0.75 against the experimental value of 0.40 . By choosing larger values of $\Gamma_{L, R}$ the theoretical model can be forced to fit the Fano factor at the expenses of overestimating the $I-V$ characteristic for about one order of magnitude. This result 

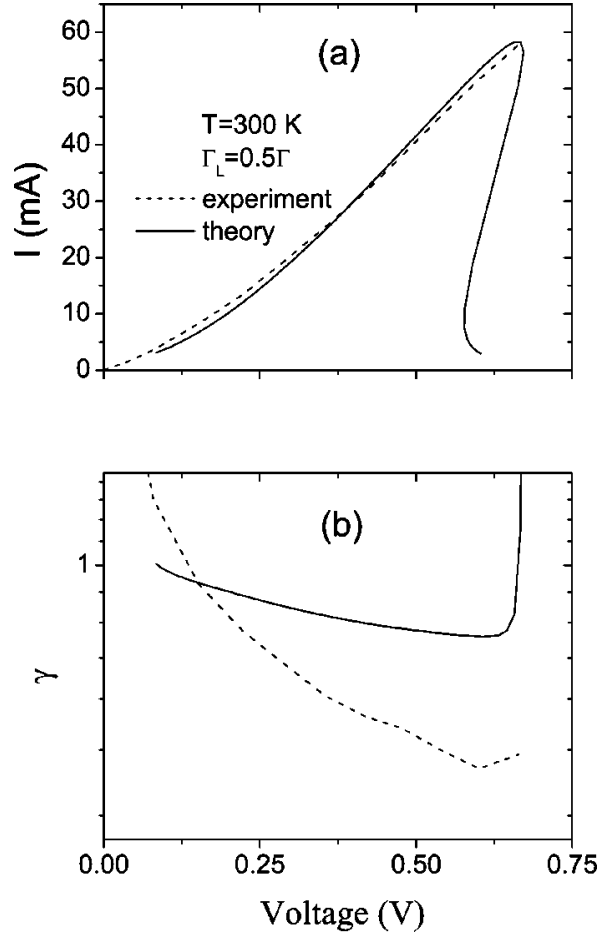

FIG. 8. Experimental and calculated dependencies of current (a) and Fano factor (b) on applied voltage for the structure in Refs. 31 and 32 at $T=300 \mathrm{~K}$.

corrects previous findings of the same authors, ${ }^{32}$ where the contribution of charge fluctuations to the total noise was underestimated. Thus, the comparison between theory and experiments at temperatures above about $77 \mathrm{~K}$ provides qualitative agreement but is not able to explain the drop of the Fano factor below 0.5 found in experiments.

\section{CONCLUSIONS}

We have implemented a quantum mechanical approach to investigate DBRDs transport and noise characteristics within the coherent tunneling regime that includes both the Pauli principle and long range Coulomb interaction. The expression for the current voltage and noise characteristics generalize previous findings..$^{25,22,26}$ In agreement with expectations, at increasing voltages theory predicts a current characteristic which exhibits a peak followed by an instability region and that before the current peak shot noise is suppressed because of the Pauli principle and/or Coulomb interaction. In addition, the theory confirms shot noise enhancement well above the full Poissonian value at the current peak as a consequence of the positive feedback between tunneling and space charge.

At low temperatures below about $4.2 \mathrm{~K}$, the suppression of shot noise starts in concomitance with the sharp increase of the current associated with the alignment within the value of $\Gamma$ of the Fermi level in the emitter with the resonant level in the quantum well. Accordingly, the Fano factor is found to exhibit a minimum near the current peak. Remarkably, the value of this minimum can be significantly below the value
0.5 of the full Poissonian value. This giant suppression of shot noise is an indication of coherent tunneling since the standard sequential tunneling can predict suppression but at most with a Fano factor not less than 0.5. At $4.2 \mathrm{~K}$, for a realistic DBRD structure we find a minumum value of the Fano factor of 0.44 that is in agreement with experiments. ${ }^{8}$ At temperatures above about $77 \mathrm{~K}$, we have found that coherent tunneling still predict that shot noise can be suppressed well below the value of 0.5 . This giant suppression has been evidenced by experiments performed at 77 and $300 \mathrm{~K}$. However, the present theory is not able to explain this noise feature with the same set of parameter values able to explain the $I-V$ characteristics. Probably other causes could lead to the suppression and further efforts are needed to provide a better interpretation of experiments.

The physical reason why shot noise suppression is expected to be more effective for coherent than for sequential tunneling is as follows. Starting from the fact that the two mechanisms responsible for shot noise suppression are the Pauli principle and Coulomb interaction, we note that both act simultaneously for coherent and sequential tunneling. Let us consider the first mechanism, which is the most relevant at low temperatures, in the case when the Fermi energy is sufficiently small so that all the electrons exhibit the same transparency. Then, coherent transport admits a transparency near equal to unity, which implies $\gamma=(1-D) \simeq 0$ according to Lesovik findings. ${ }^{34}$ For sequential transport the total transparency is always less than unity because of the finite value of the differential rates controlling the relaxation of carrier number fluctuations inside the two terminal device through the contacts. As a consequence, under coherent transport for the possible case of full transparency (i.e., $D=1$ ) there is no noise. By contrast, under sequential transport the presence of scattering inside the quantum well always introduces noise. This example illustrates why the Pauli principle is more efficient in suppressing shot noise under coherent than sequential transport. By considering Coulomb interaction, which is more relevant at high temperatures, we recall that in the absence of collisions it provides giant shot noise suppression ${ }^{36}$ as in the vacuum tubes ${ }^{37}$ because electron reflection in this case is due only to Coulomb interaction. It is clear that the presence of scattering provides additional random mechanisms for electrons returning to the emitter and, therefore, provides an additional source of noise. Even this example shows that Coulomb interaction is more efficient in suppressing shot noise under coherent than sequential transport. We finally want to stress that the main reason of the difference between these approaches stems from the fact that the sequential tunneling is based on a master equation ${ }^{35,11}$ for treating fluctuations of carrier numbers inside the quantum well. As a consequence, its intrinsic limit coincides with that of two independent resistors (or vacuum diodes) connected in series and each of them exhibiting full shot noise. This system yields a maximum suppression of shot noise down to the value of 0.5 . By contrast, partition noise, inherent to a quantum coherent formalism, can be fully suppressed down to zero in the presence of a fully transparent barrier and weak Coulomb interaction like in vacuum diodes. 


\section{ACKNOWLEDGMENTS}

The authors thank Professor V. Volkov of Moscow Institute of Radioengineering and Electronics for having suggested the problem of this research and Professor M. Büttiker of Geneva University for having addressed the importance of charge fluctuations in the calculation of the current fluctuations. This research has been performed within the project "Noise models and measurements in nanostructures" supported by the Italian Ministery of Education, University and Research (MIUR) through the cofin03. Partial support from the Italian Ministry of Foreign Affairs through the Volta Landau Center, and the SPOT-NOSED Project No. IST-2001-38899 of the EC is gratefully acknowledged.

\section{APPENDIX}

Here we detail the calculations to evaluate the current operator, the differential capacitances of the DBRDs and the solution of the Schrödinger equation for the voltage dependence of the first electron level in the emitter.

\section{A. Current operators}

By using Eqs. (3)-(5) and (7), the explicit expression for $A_{\beta, \gamma}^{\alpha}\left(E_{\beta}, E_{\gamma}\right)$ is the following:

$$
A_{\beta, \gamma}^{\alpha}\left(E_{\beta}, E_{\gamma}\right)=-2 \pi i\left[\frac{T_{\alpha} T_{\beta}^{*} B_{\alpha \gamma}\left(E_{\gamma}\right)}{E_{\beta}-E_{r}-i \Gamma / 2}-\frac{T_{\alpha}^{*} T_{\gamma} B_{\alpha \beta}^{*}\left(E_{\beta}\right)}{E_{\gamma}-E_{r}+i \Gamma / 2}\right],
$$

where

$$
B_{\alpha \beta}\left(E_{\beta}\right)=\left(\delta_{\alpha \beta}-i \pi \frac{T_{\alpha}^{*} T_{\beta} \rho_{\alpha}}{E_{\beta}-E_{r}+i \Gamma / 2}\right) .
$$

From earlier, we note the following properties:

$$
\begin{aligned}
A_{L L}^{L}(E, E) \rho_{L} & =A_{R R}^{R}(E, E) \rho_{R}=-A_{R R}^{L}(E, E) \rho_{R} \\
& =-A_{L L}^{R}(E, E) \rho_{L}=D(E)
\end{aligned}
$$

and

$$
\begin{aligned}
A_{L R}^{L}(E, E) A_{R L}^{L}(E, E) \rho_{L} \rho_{R} & =A_{L R}^{R}(E, E) A_{R L}^{R}(E, E) \rho_{L} \rho_{R} \\
& =D(E)[1-D(E)] .
\end{aligned}
$$

Equations (A3) and (A4) are useful for current and noise calculations.

\section{B. Capacitances}

By recalling that $Q_{L}=-\kappa \varphi_{L}^{\prime} / 4 \pi$ from Eq. (12) we have

$$
\begin{aligned}
C_{e}\left(u_{L}\right)= & q\left[N_{c} F_{1 / 2}\left(\frac{F_{L}+q u_{L}}{k_{B} T}\right)-n\right] \sqrt{\frac{\kappa}{8 \pi k_{B} T}} \\
& \times\left[N_{c} F_{3 / 2}\left(\frac{F_{L}+q u_{L}}{k_{B} T}\right)\right. \\
& \left.-N_{c} F_{3 / 2}\left(\frac{F_{L}}{k_{B} T}\right)-\frac{q}{k_{B} T} n u_{L}\right]^{-1 / 2} .
\end{aligned}
$$

If the electron accumulation is relevant (i.e., $q u_{L}>k_{B} T$ ) then we can write

$$
C_{e}\left(u_{L}\right) \approx q \frac{N_{c} F_{1 / 2}\left(\frac{F_{L}+q u_{L}}{k_{B} T}\right)}{\sqrt{\frac{8 \pi k_{B} T}{\kappa \kappa_{0}} N_{c} F_{3 / 2}\left(\frac{F_{L}+q u_{L}}{k_{B} T}\right)}} .
$$

By substituting for $Q_{R}$ the value given in Eq. (15), for $C_{c}$ we find

$$
\begin{aligned}
C_{c}= & q\left[n-N_{c} F_{1 / 2}\left(\frac{F_{L}+q u_{L}-q V}{k_{B} T}\right)\right] \sqrt{\frac{\kappa}{8 \pi k_{B} T}} \\
& \times\left[N_{c} F_{3 / 2}\left(\frac{F_{L}+q u_{R}-q V}{k_{B} T}\right)\right. \\
& \left.-N_{c} F_{3 / 2}\left(\frac{F_{L}-q V}{k_{B} T}\right)-\frac{q n}{k_{B} T}\left(u_{R}-V\right)\right]^{-1 / 2} .
\end{aligned}
$$

If $q\left(V-u_{R}\right) /\left(k_{B} T\right)>1$, then we have the usual expression for the capacitance of the depletion region

$$
C_{c} \approx \sqrt{\frac{q \kappa n}{8 \pi\left(V-u_{R}\right)}} .
$$

\section{Energy of the first electron level in the emitter}

The one dimensional Schrödinger equation for an electron moving in the potential $-q \varphi(x)$ can be written in the following form:

$$
\frac{d y}{d x}+y^{2}+\frac{2 m}{\hbar^{2}}(q \varphi+\varepsilon)=0
$$

here $y=\Psi^{\prime} / \Psi, \Psi$ is the electron wave function and $\varepsilon$ the electron energy. Since $d \varphi / d x$ in the emitter is function of $\varphi$ [see Eqs. (11) and (12)], instead of $x$ it is convenient to use $\varphi$ asvariable quantity so that

$$
\frac{d y}{d \varphi} \frac{d \varphi}{d x}+y^{2}+\frac{2 m}{\hbar^{2}}(q \varphi+\varepsilon)=0
$$

where

$$
\begin{aligned}
\frac{d \varphi}{d x}= & \sqrt{\frac{8 \pi k_{B} T}{\kappa}}\left[N_{c} F_{3 / 2}\left(\frac{F_{L}+q \varphi}{k_{B} T}\right)\right. \\
& \left.-N_{c} F_{3 / 2}\left(\frac{F_{L}}{k_{B} T}\right)-\frac{q}{k_{B} T} n \varphi\right]^{1 / 2} .
\end{aligned}
$$

The potential $\varphi$ in the emitter takes values in the range from 0 far from the barriers $(x \rightarrow-\infty)$ to $u_{L}$ on the border of the left barrier. We note that far from the barriers the potential falls exponentially $\varphi \sim \exp \left(x / \lambda_{D}\right)$ when $q \varphi \ll k_{B} T$, where $\lambda_{D}$ is the Debye screening length. For localized state the electron wave function $\Psi \sim \exp \left(k_{1} x\right)$ for $x \rightarrow-\infty$, where $k_{1}=\sqrt{-2 m \varepsilon} / \hbar$ and, thus, we have as boundary condition $y(\varphi=0)=k_{1}$. Since we suppose that the field in the barrier is absent, the electron wave function in the barrier is 
$\Psi \sim \exp \left(-k_{2} x\right)$ where $k_{2}=\sqrt{2 m\left(\Delta E_{c}-q u_{L}-\varepsilon\right)} / \hbar$ and $\Delta E_{c}$ is the conduction band offset on the barrier border. By using the conditions of continuity of the wave function and of its derivative, we obtain the second boundary condition
$y\left(\varphi=u_{L}\right)=-k_{2}$. Equations (C2) and (C3) together with the boundary conditions allow us to find the energy of the first electron level in the potential well of the emitter by numerical calculations.
${ }^{1}$ R. Tsu and L. Esaki, Appl. Phys. Lett. 22, 562 (1973).

${ }^{2}$ V. Goldman, D. Tsui, and J. Cunningham, Phys. Rev. Lett. 58, 1256 (1987).

${ }^{3}$ Y. M. Blanter and M. Büttiker, Phys. Rep. 336, 1 (2000).

${ }^{4}$ L. L. Chang, L. Esaki, and R. Tsui, Appl. Phys. Lett. 24, 593 (1974).

${ }^{5}$ S. Luryi, Appl. Phys. Lett. 47, 490 (1985).

${ }^{6}$ Y. P. Li, A. Zaslavsky, D. C. Tsui, M. Santos, and M. Shayegan, Phys. Rev. B 41, 8388 (1990).

${ }^{7}$ L. Y. Chen and C. S. Ting, Phys. Rev. B 43, 4534 (1991).

${ }^{8}$ E. R. Brown, IEEE Trans. Electron Devices 39, 2686 (1992).

${ }^{9}$ S. Hershfield, Phys. Rev. B 46, 7061 (1992).

${ }^{10}$ E. Runge, Phys. Rev. B 47, 2003 (1993).

${ }^{11}$ G. Iannaccone, M. Macucci, and B. Pellegrini, Phys. Rev. B 55, 4539 (1997).

${ }^{12}$ H. B. Sun and G. Milburn, Phys. Rev. B 59, 10748 (1999).

${ }^{13}$ A. Przadka, K. J. Webb, D. B. Janes, H. C. Liu, and Z. R. Wasilewski, Appl. Phys. Lett. 71, 530 (1997).

${ }^{14}$ V. Kuznetsov, E. Mendez, J. Bruno, and J. Pham, Phys. Rev. B 58, R10159 (1998).

${ }^{15}$ J. C. Egues, S. Hershfield, and J. V. Wilkins, Phys. Rev. B 49, 13517 (1994).

${ }^{16}$ M. Jahan and A. Anwar, Solid-State Electron. 38, 429 (1995).

${ }^{17}$ L. Chen and C. Ting, Phys. Rev. B 46, 4714 (1992).

${ }^{18}$ J. Davies, P. Hyldgaard, S. Hershfield, and J. Wilkins, Phys. Rev. B 46, 9620 (1992).

${ }^{19}$ L. Chen, Phys. Rev. B 48, 4914, (1993).

${ }^{20}$ K. Hung and G. Wu, Phys. Rev. B 48, 14687 (1993).

${ }^{21}$ S. Hershfield, J. H. Davies, P. Hyldgaard, C. J. Stanton, and J. W. Wilkins, Phys. Rev. B 47, 1967 (1993).
${ }^{22}$ Y. M. Blanter and M. Büttiker, Phys. Rev. B 59, 10217 (1999).

${ }^{23}$ V. Ya. Aleshkin and L. Reggiani, Phys. Rev. B 64, 245333 (2001).

${ }^{24}$ V. Ya. Aleshkin, L. Reggiani, and A. Reklaitis, Phys. Rev. B 63, 085302 (2001).

${ }^{25}$ D. V. Averin, J. Appl. Phys. 73, 2593 (1993).

${ }^{26}$ Y. Wei, B. Wang, J. Wang, and H. Guo, Phys. Rev. B 60, 16900 (1999).

${ }^{27}$ N. Ashcroft and N. Mermin, Solid State Physics (Holt, Rinehart and Winston, New York, 1976).

${ }^{28}$ J. M. L. Figueiredo, C. R. Stanley, A. R. Boyd, C. N. Ironside, S. G. McMeekin, and A. M. P. Leite, Appl. Phys. Lett. 74, 1197 (1999).

${ }^{29}$ T. Martin and R. Landauer, Phys. Rev. B 45, 1742 (1992).

${ }^{30}$ H. Nyquist, Phys. Rev. 32, 110 (1925).

${ }^{31}$ N. V. Alkeev, V. E. Lyubchenko, C. N. Ironside, J. M. L. Figueiredo, and C. R. Stanley, J. Commun. Technol. Electron. 47, 228 (2002).

${ }^{32}$ V. Ya. Aleshkin, L. Reggiani, N. V. Alkeev, V. E. Lyubchenko, C. N. Ironside, J. M. L. Figuiredo, and C. R. Stanley, Semicond. Sci. Technol. 18, L35 (2003).

${ }^{33}$ N. V. Alkeev, V. E. Lyubchenko, C. N. Ironside, S. G. McMeekin, and A. M. P. Leite, J. Commun. Technol. Electron. 45, 911 (2000).

${ }^{34}$ G. B. Lesovik, JETP Lett. 49, 592 (1989).

${ }^{35}$ J. H. Davies, J. C. Egues, and J. W. Wilkins, Phys. Rev. B 52, 11259 (1995).

${ }^{36}$ G. Gomila, I. R. Cantalapiedra, T. Gonzalez, and L. Reggiani, Phys. Rev. B 66, 075302 (2002).

${ }^{37}$ A. Van der Ziel, Noise (Prentice Hall, New York, 1954). 